\title{
Health, stress and welfare aspects of fitting research devices to vultures and condors
}

\section{Neil A. Forbes BVetMed DipECZM (avian) FRCVS}

RCVS Recognised Specialist in Zoo and Wildlife (avian) veterinary medicine http://dx.doi.org/10.4314/vulnew.v78ai1.3
The following principles should guide the fitting of research devices to vultures and condors. First, do no harm and second - trap, catch, restrain and fit tags / transponders in a 'welfare sound manner' in order to gain accurate research data in an ethical way

Operators may feel reassured that they have not observed any obvious signs of distress during the deployment of research devices, but even slight stress can result in suppression of the immune system, which will then render the bird more susceptible to infections, in particular viral or fungal (e.g. Aspergillus spp.) infections, which are at best debilitating and frequently fatal, with the effects often not being seen until 4-6 weeks later. Avoiding or minimising stress is therefore vitally important.

Birds do not sweat and heat loss by panting is limited, especially if the head is restrained, covered or hooded. The normal core body temperature of birds is $40-41^{\circ} \mathrm{C}\left(104-111.2^{\circ} \mathrm{F}\right)$. An increase of $4^{\circ} \mathrm{C}$ $\left(7^{\circ} \mathrm{F}\right)$ to a bird's core body temperature is inevitably fatal.

The duration of the procedure, macro- and micro-environmental ambient temperature, restraint and management, time of day and use of restraint boxes to hold birds in, all have a bearing and must be correctly managed and monitored.

Operators should not deploy devices on birds, unless they can be confident of completing the task (catch to release) within 15 minutes. Preparing everything in advance is essential. Practice on soft toys followed by cadavers is essential prior to harnessing live birds.

Planning should include ensuring that the procedure is carried out at a cool time of year or day. Harnessing at temperatures over $26^{\circ} \mathrm{C}$ carries significant risk and should not be conducted if over $30^{\circ} \mathrm{C}$. When trapping, netting or catching a number of birds to harness, any birds awaiting harnessing must be kept in a cool environment pending harnessing. Being enclosed in carrier boxes with no internal ventilation results in the bird heating up rapidly, even when in the shade. During device fitting application, the bird must never be laid on a hot or warm surface, (e.g. on your lap, against your chest or on a warm table or rock), cool bag frozen blocks, or self cooling gel filled pads for dog beds ${ }^{\mathrm{a}}$, can be very useful, but you must be careful to avoid perforating the gel pads.

\section{Monitoring during device deployment}

One member of the team must be delegated the task of observing throughout the procedure for any signs of distress; collapse (abnormal respiratory sounds, reactions, nervous signs (e.g. tremors, shaking etc.)), gasping, breathing more deeply, change in vocalisation, becoming limp, feet feeling or measuring hot. That person must have the power to abort the procedure at any point.

It is useful and important to keep monitoring the bird's temperature. The most accurate method is to use a cloacal probe, but this must be soft (such that internal trauma is impossible) and the readout must be remote from the body. Some inexpensive types of digital indoor thermometer/hygrometer with a probe sensor can be used ${ }^{\mathrm{b}}$. Alternatively, one can use a remote infrared laser thermometer ${ }^{\mathrm{c}}$ to record the skin temperature of the feet. Do not measure facial temperature for fear of laser damage to the retina. Temperature should be checked every 10 minutes, increasing to every 5 minutes if it has already increased by $2^{\circ} \mathrm{C}$ or $3.5^{\circ} \mathrm{F}$ ). If core body 
temperature increases by $3.5^{\circ} \mathrm{C}$ or $6^{\circ} \mathrm{F}$, or skin temperature increases by $4^{\circ} \mathrm{C}$ or $7^{\circ} \mathrm{F}$, the procedure must be aborted immediately and restraint of the bird released as soon as possible. The body skin, feet, face and neck can be cooled by wetting with water. As the water evaporates, the body will cool.

\section{Impaired breathing}

Overheating apart, birds do not have a diaphragm, as such, greater care must be taken not to compromise their breathing. A bird should never be kept on its back as this will result in a 10-60\% reduction in tidal volume. Care must be taken not to squash or constrict the trachea when restraining the head. No jacket or cloth should be wrapped tight around the body and no operator or assistant should restrict respiration by holding around the chest.

\section{To hood or not to hood?}

Keeping a bird in the dark by hooding or using a lightweight dark cloth around the head, will generally keep a diurnal bird calmer, however great care must be taken to ensure that if a bird regurgitates, there is no risk of aspiration into the air sacs or lungs. Therefore any hood applied should be loose-fitting, facilitating good beak opening and unhindered regurgitation. Whilst hooding does reduce heart and respiratory rate, it does not reduce increases in body temperature. It is also important to appreciate that hooding does impair visual monitoring for signs of distress by the operator.

\section{Safety considerations when mounting research devices on the wing}

Most of the methods used to attach research devices to vultures and condors do not involve any piercing of the birds' tissues. However, the attachment of coloured plastic or fabric tags bearing alphanumeric characters, without a device, to the wing with a pin or peg through the propatagium is a widely-used method for marking vultures and condors as individuals. The propatagium is an elastic membrane extending between the shoulder and carpus. It forms the leading edge of the wing and is vital for aerodynamics required for flight. This region is not simply skin, but contains vital tendons and ligaments, with feather follicles and blood vessels distributed throughout. It is possible to visualise and feel the locations of feather follicles and blood vessels if surgical alcohol is applied to the ventral aspect of the propatagium. Misplacement of the tag can and does cause damage to the vital structures resulting in crippling injuries, grounding and loss of life if the bird is not rescued.

This problem has been documented in a recent review of eight grounded and failed long-term released rehabilitated Gyps vultures marked with identification tags which had been positioned incorrectly (Hirschauer et al. 2019). Dissection of vulture cadavers revealed the vital structures which must be avoided during propatagial wing tag placement. Figure A.1 shows the location and dimensions of the safe area of the propatagium of the Cape Vulture Gyps coprotheres within which the pin or peg of the tag and any washer should be placed. The structures to be avoided are the same in other vulture species, but the dimensions of the safe area and its margins would need to be adjusted to allow for differences in body size. Hirschauer et al. (2019) found that placement of the pin or peg of the tag outside the safe area in any direction would cause injury. These recommendations have been determined for identification tags without research devices, but tracking devices weighing up to $73 \mathrm{~g}$ have also been attached to the propatagium of Gyps vultures and condors (see Gilbert et al. (2007) and Method 6 of this guide). The same safety 
considerations for the placement of the pin or peg also apply to such deployments (Figure A.1).

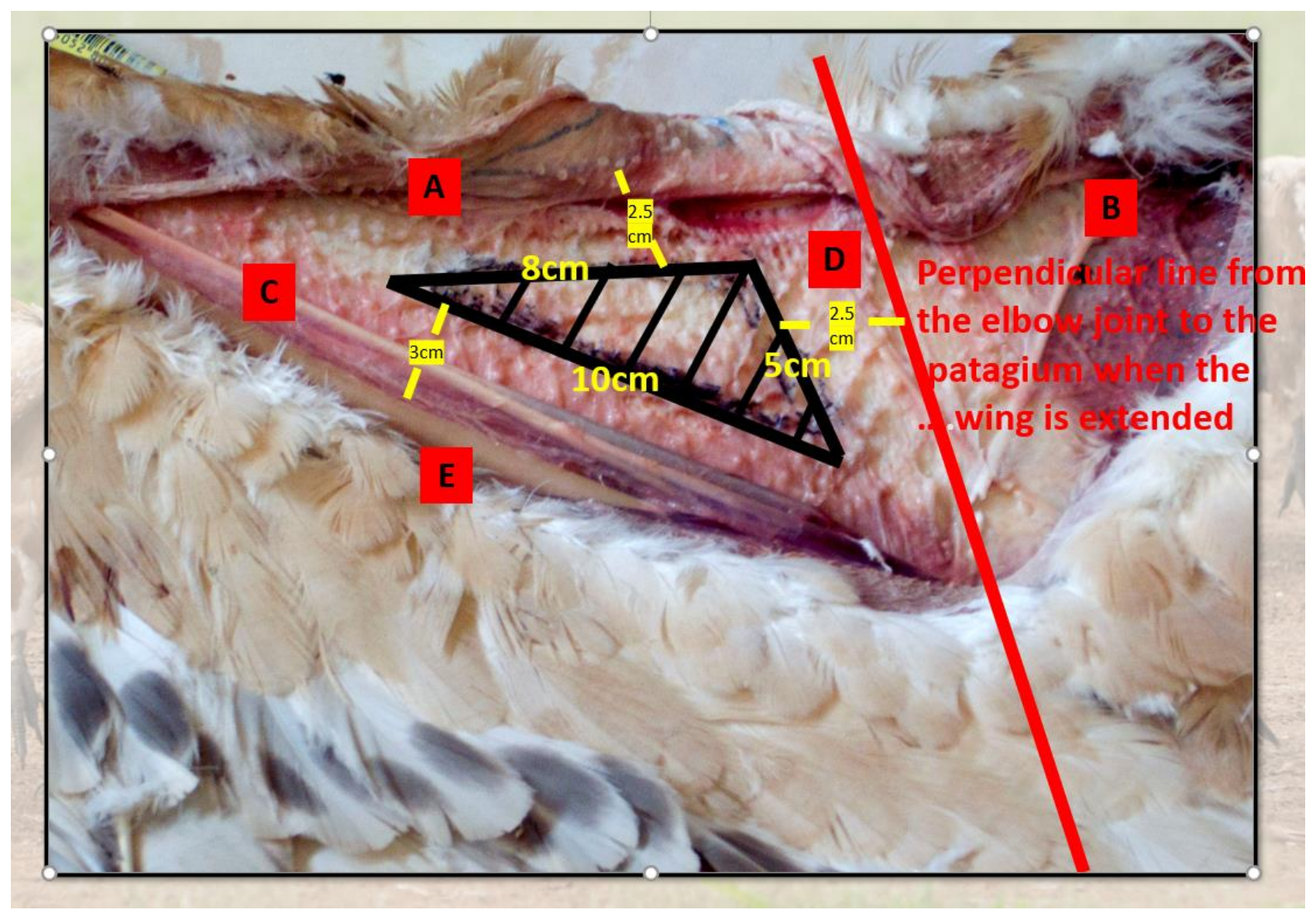

Figure A1: Ventral view of the safe area for wing tag placement in the Cape vulture. The underside of the extended right wing is shown with the proximal (closest to body) part of the wing on the right. The red line is perpendicular to the extended wing and extends from the elbow joint (bottom of photo) to the bend in the leading edge of the propatagium (top of photo). The triangular safe area for insertion of the pin or peg of a propatagial tag is shown by the black outline and hatching. The safe area commences $2.5 \mathrm{~cm}$ distal (towards the carpus) from the red line. The cranial limitation of the safe area is $2.5 \mathrm{~cm}$ caudal to (behind) the leading edge of the wing, and the caudal extremity of the safe area is $3 \mathrm{~cm}$ cranial to (in front of) the radius. Important structures to be avoided are marked by the letters in red boxes: (A) Ligamentum propatagialis pars longus, (B) Ligamentum propatagialis pars brevis, (C) $M$. extensor carpi radialis (running in parallel to the radius and ulna bones) (D) Ligamentum limitans cubiti, and (E) radius (bone).

\section{On completion of device fitting}

If it is possible to observe the bird in a confined area (e.g. travel box) on completion of harnessing to ensure the bird can stand, walk freely and appears clinically normal, this is advisable. Any bird whose procedure has extended beyond 20 minutes, whose core body temperature has increased above $44^{\circ} \mathrm{C}$ $\left(111^{\circ} \mathrm{F}\right)$, who looks distressed, is not behaving normally, who is breathing faster than normal, whose eyes are lemon shaped and sleepy rather than round and bright, should have oral fluid therapy 
administered, so long as the operator is suitably experienced and equipped to administer such. Fluids should be administered at a rate of $20 \mathrm{ml} / \mathrm{kg}$ body weight, taking great care to administer into the oesophagus and not the trachea, using one of several suitable syringes, a metal $12-6 \mathrm{~g}$ metal feeding tube, or a $6-10 \mathrm{~mm}$ diameter plastic tube (so long as a wooden or plastic gag is used to prevent this from being chewed off. Glucose saline or Lactated Ringers solution should be administered which are available as concentrated solutions to dilute, as powders to dissolve or as ready to use fluid bags.

\section{Take-home points:}

(1) Placing the bird's head in the dark may assist.

(2) Minimising abnormal sounds/noise whilst birds are close to us.

(3) Prepare everything well in advance.

(4) Minimise duration of boxing, restraint and handling.

(5) Be confident and quick when catching a bird.

(6) Minimise the risk of overheating, if it is unavoidable consider the involvement of a vet and sedation for the bird (which does reduce overheating) - always reverse sedation prior to release.

(7) Be aware of and monitor for effects of stress or collapse (abnormal sounds, reactions, nervous signs, gasping, breathing more deeply, change in vocalisation, becoming limp, feet feeling or measuring hot).

(8) Avoid harnessing in hot conditions $\left(>30^{\circ} \mathrm{C},>86^{\circ} \mathrm{F}\right)$.

(9) Practice (e.g. on a dead bird) several times beforehand so your technique is as quick and skilled as possible.

(10) Restrain the bird correctly to avoid causing respiratory distress or overheating.

(11) Monitor the bird's temperature. Record the temperature each time. If the temperature goes up more than the specified amount, spray feet, face and crop with water or abort the procedure.

(12) When the harness is on, you should put the bird in a box and watch it through a hole to check that it stands and walks normally.

(13) All operators should carry with them a suitable first aid kit and be equipped and be experienced in administering oral fluid therapy if relevant, prior to release of the bird.

\section{Products mentioned}

${ }^{a}$ XIAPIA Cooling Mat for Dogs Large Gel Pet Cool Mat Self Cool Pad Waterproof and Scratch Resistant for Dogs and Cats:

https://tinyurl.com/y43qogep

${ }^{\mathrm{b}}$ Digital Indoor Thermometer/Hygrometer: https://tinyurl.com/y42waw5r

'Etekcity Lasergrip 774 Non-contact Digital Laser IR Infrared Thermometer: https://www.amazon.co.uk/dp/B01AT9TM3M?tag=celheal-21 